\title{
WGA is a probe for migrasomes
}

\author{
Lilian Chen ${ }^{1,3}$, Liang $\mathrm{Ma}^{1,2,3}$ and $\mathrm{Li} \mathrm{Yu^{1 }}$
}

\section{Dear Editor,}

During cell migration, cells leave behind long tubular retraction fibers. Previously, we identified that vesicles with diameters up to $2 \mu \mathrm{m}$, which we called migrasomes, grow at the tips and intersections of retraction fibers ${ }^{1}$. We found that the migrasomes contain cytosol, which can be released into the extracellular environment or directly taken up by surrounding cells ${ }^{1}$. Therefore, we proposed that migrasomes may mediate cell-cell communications. We identified Tetraspanin4 (Tspan4) as a marker for migrasomes, and thus we can detect migrasomes in various types of cells and characterize the process of migrasome biogenesis. However, the limitations of using a fluorescently tagged marker protein for detection of an organelle are obvious. First, it is time consuming; transfection and expression of the marker protein takes at least 1 day before observation. Second, the transfections of many cells, especially many primary cells, are very difficult. For these cells, complicated transfection strategies (e.g., virus-mediated transfection) are required, which further increase the time and cost of migrasome detection. Finally, overexpression of fluorescently tagged protein always has the risk that the overexpressed marker protein may change the biogenesis of migrasomes, thus causing artifacts. Thus a rapid, easy, and non-interfering method is needed for detection of migrasomes.

By serendipity, we found that fluorescently tagged wheat-germ agglutinin (WGA), a lectin that binds specifically to sialic acid and $N$-acetyl-D-glucosamine ${ }^{2}$, labeled migrasomes in living cells (Fig. 1a). Moreover, florescence intensity analysis showed that the WGA signal on migrasomes was much higher than the WGA signal on

\footnotetext{
Correspondence: Liang Ma (liangm92@gmail.com) or Li Yu (liyulab@mail. tsinghua.edu.cn)

${ }^{1}$ State Key Laboratory of Membrane Biology, Tsinghua University-Peking University Joint Center for Life Sciences, School of Life Sciences, Tsinghua University, 100084 Beijing, China

${ }^{2}$ Department of Molecular and Cellular Biology, Howard Hughes Medical Institute, University of California, Berkeley, CA 94720, USA

Full list of author information is available at the end of the article.

These authors contributed equally: Lilian Chen, Liang Ma
}

retraction fibers (Fig. 1b, c), indicating that WGA prefers to bind to migrasomes. Finally, WGA effectively labeled migrasomes in all the cell lines we tested (Supplementary Fig. S1). Thus fluorescently tagged WGA can be used as a probe for migrasomes.

Next, we investigated the optimal conditions for WGA staining of fixed cells. We fixed cells in paraformaldehyde (PFA), methanol, and glutaraldehyde (GA), then stained them with WGA. Migrasomes and retraction fibers could be preserved and labeled efficiently in GA-fixed cells (Fig. 1d); however, cells generated strong autofluorescence after GA fixation, which could be quenched effectively by treating the cells with sodium borohydride after fixation (Supplementary Fig. S2a). In contrast, in methanol- or PFA-fixed cells, retraction fibers and migrasomes were not preserved well (Fig. 1d). In GAfixed cells, we found that the optimum working concentration of WGA for migrasome staining was $1 \mu \mathrm{g} / \mathrm{ml}$ (Supplementary Fig. S2b). Using this concentration, staining for as short as $1 \mathrm{~min}$ generated a strong signal (Supplementary Fig. S2c).

Next, we tested whether WGA staining can be used in time-lapse imaging. First, we stained cells with WGA, then removed the medium and washed cells with phosphate-buffered saline to reduce background fluorescence. We found that most of the WGA signal was endocytosed into the cells $1 \mathrm{~h}$ after labeling (Supplementary Fig. S3a). Thus this approach is not suitable for time-lapse imaging $>1 \mathrm{~h}$. To monitor migrasome formation for longer periods of time, we kept WGA in the culture medium during imaging. We found that this approach gave a reasonable signal-to-noise ratio and enabled us to monitor migrasomes for a much longer time (Fig. 1e). To check whether long-term exposure to WGA can affect migrasome biogenesis and cell migration, we performed time-lapse imaging and compared the migration of cells with or without WGA. We found that, although the presence of WGA slightly affected cell migration (Supplementary Fig. S3b), however, the formation of migrasomes was not affected by the presence of 


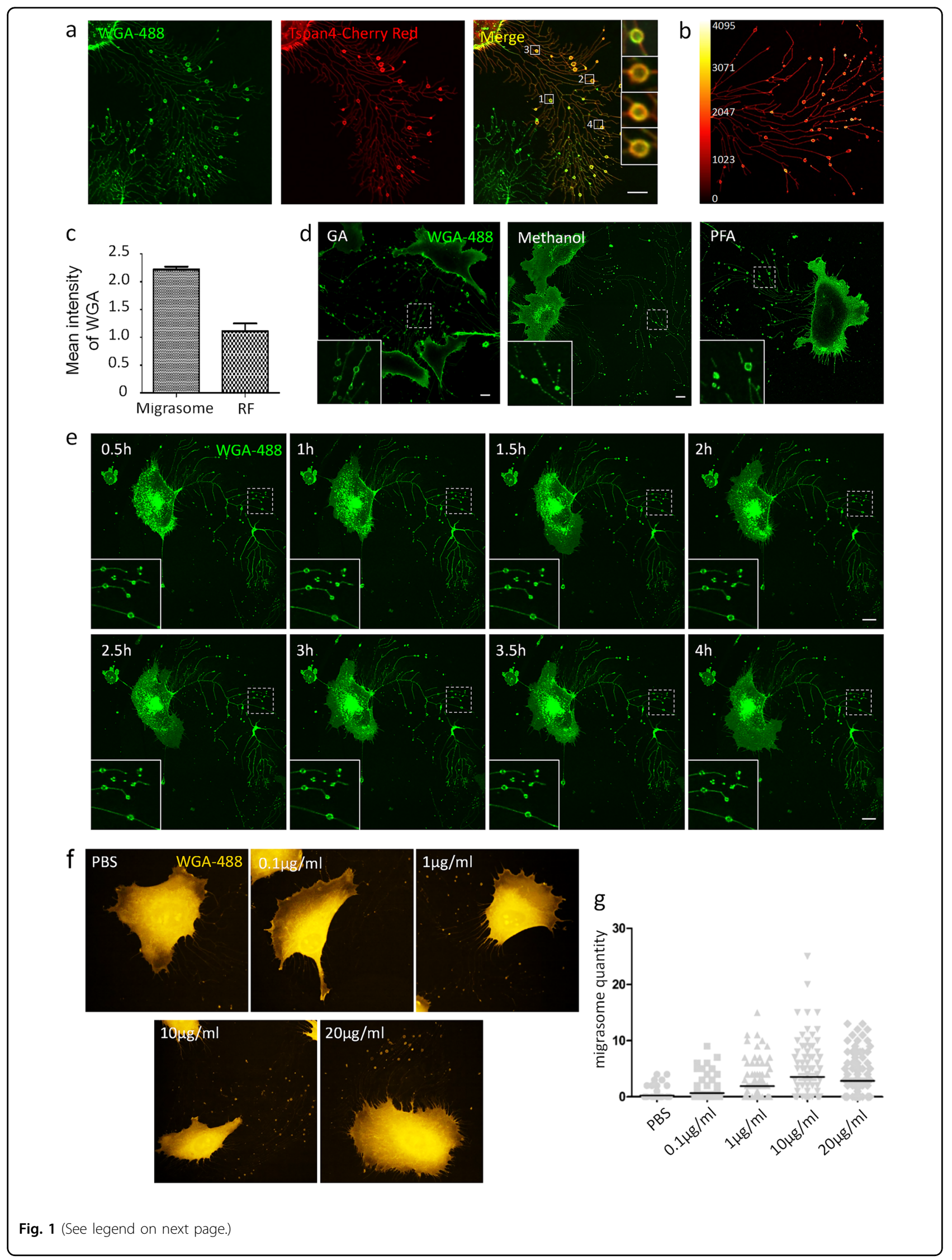


(see figure on previous page)

Fig. 1 Detection of migrasomes by WGA. a L929 cells were transfected with Tspan-mCherry and cultured for $12 \mathrm{~h}$, then stained with $1 \mu \mathrm{g} / \mathrm{ml}$ WGA-

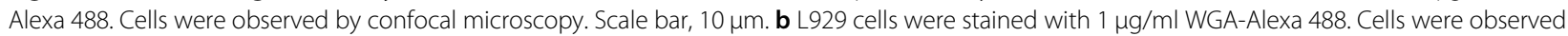
by confocal microscopy. Enrichment of WGA-Alexa 488 on migrasomes was revealed by fluorescence intensity heat mapping. c Cells from a were measured for WGA-Alexa 488 fluorescence intensity in migrasomes and retraction fibers by the Olympus FLUOVIEW Ver.2.0b Viewer software; $n=450$ migrasomes/retraction fibers (RFs) from three independent experiments. $t$ test, $P<0.0001$. Error bars indicate mean \pm SD. $\mathbf{d} L 929$ cells were fixed with $2.5 \%$ glutaraldehyde, methanol, or $4 \%$ paraformaldehyde, then stained with WGA-Alexa 488. Cells were observed by confocal microscopy. Scale bar, $10 \mu \mathrm{m}$. e $\mathrm{L} 929$ cells were stained with $1 \mu \mathrm{g} / \mathrm{ml}$ WGA-Alexa 488 , which was present in the medium during imaging. Images were captured every 30 min for $4 \mathrm{~h}$. f $\mathrm{L929}$ cells were cultured for $12 \mathrm{~h}$ on microwell plates coated with different concentrations of fibronectin, then fixed with $2.5 \%$ glutaraldehyde and stained with WGA. Images were taken with the Opera Phenix system. $\mathbf{g}$ The number of migrasomes per cells with different concentrations of fibronectin in $\mathbf{f}$

WGA (Supplementary Fig. S3c). Finally, we tested whether WGA staining is compatible with a high-content screening system. Previously, we showed that fibronectin increases the formation of migrasomes; however, the optimal concentration of fibronectin is not known yet. By WGA labeling, we were able to test a series of concentrations using an Opera Phenix high-content screening system, and we determined that $10 \mu \mathrm{g} / \mathrm{ml}$ fibronectin is the optimal working concentration for generation of migrasomes (Fig. 1f, g).

In summary, we found that WGA is a probe for convenient, rapid detection of migrasomes in both fixed and living cells.

\section{Acknowledgements}

We are grateful to Nikon Instruments (Shanghai) and the Tsinghua Cell Biology Core Facility for providing technical support. This research was supported by the Ministry of Science and Technology of the People's Republic of China (2016YFA0500202 and 2017YFA0503404), the National Natural Science Foundation of China (31790402, 31430053, and 31621063), Natural Science Foundation of China international cooperation and exchange program (31561143002), and Beijing Advanced Innovation Center for Structural Biology, Tsinghua University.

\section{Author contributions}

L.M. and L.C. performed the majority of the experiments. L.Y. and L.M. wrote the manuscript.

\section{Author details}

'State Key Laboratory of Membrane Biology, Tsinghua University-Peking University Joint Center for Life Sciences, School of Life Sciences, Tsinghua University, 100084 Beijing, China. ${ }^{2}$ Department of Molecular and Cellular Biology, Howard Hughes Medical Institute, University of California, Berkeley, CA 94720, USA. ${ }^{3}$ Present address: Department of Molecular and Cell Biology, University of California, Berkeley, CA 94720, USA

\section{Conflict of interest}

The authors declare that they have no conflict of interest.

\section{Publisher's note}

Springer Nature remains neutral with regard to jurisdictional claims in published maps and institutional affiliations.

Supplementary Information accompanies the paper at (https://doi.org/ 10.1038/s41421-018-0078-2).

Received: 26 August 2018 Revised: 12 December 2018 Accepted: 17 December 2018

Published online: 26 February 2019

\section{References}

1. Ma, L. et al. Discovery of the migrasome, an organelle mediating release of cytoplasmic contents during cell migration. Cell Res. 25, 24-38 (2015).

2. Monsigny, M., Roche, A. C., Sene, C., Maget-Dana, R. \& Delmotte, F. Sugar-lectin interactions: how does wheat-germ agglutinin bind sialoglycoconjugates? Eur. J. Biochem. 104, 147-153 (1980). 